\title{
THE ROTARY ELECTRORHEOLOGICAL EFFECT
}

\author{
THOMAS C. HALSEY \\ Exxon Research and Engineering, Route 22 East \\ Annandale, NJ 08801, U.S.A. \\ and \\ ROBERT A. ANDERSON and JAMES E. MARTIN \\ Advanced Materials Physics Division, Sandia National Laboratories \\ Albuquerque, NM 87185, U.S.A.
}

\begin{abstract}
The viscous response of electrorheological fluids is usually manipulated through the use of DC or uniaxial AC electric fields. The result is that fibrillated structures parallel to the field form in a quiescent fluid; the distortion of such structures in a flow determines the enhanced viscous response, at least at low and moderate flow rates. We have conducted preliminary studies of electrorheological response in a different field configuration-a rotating electric field. With respect to the uniaxial AC case, there are two new developments in this type of field. The structures formed are disk-like, in the plane of the rotating field. Furthermore, the structures rotate either with or against the field, depending on the dielectric or conductivity contrast with the surrounding fluid.
\end{abstract}

\section{Introduction}

At the heart of the electrorheological (ER) effect is the ability to manipulate the interactions between colloidal particles through an applied electric field. While there has been considerable study of the way in which particle and fluid composition and response influence the ER response of the colloidal system ${ }^{1}$, study of the influence of the electric field has been for the most part restricted to a study of the influence of the driving frequency of a uniaxial field.

In this contribution, it is our purpose to demonstrate that interesting effects arise by using more complicated electric field configurations. We focus upon circularly polarized electric fields. If the rotation frequency of the field is significantly lower than the characteristic response frequency of the polarization mechanism for the particles, then the interactions of the particles, while still uniaxial, are qualitatively reversed with respect to the normal ER case-particles attract if they are in the plane of the electric field, and repel along the direction perpendicular to this plane. This results in the formation of disk-like structures: we propose to call this the "Rotary" ER effect.

\section{MASTER}


Any polarization mechanism will display some phase lag with the applied electric field. In the ordinary ER effect, the polarization is approximately collinear with the field even for appreciable phase lag, so while the strength of the ER effect may be altered by this phase lag, no torques develop on the particles. In the rotary ER effect, such torques play a significant role, and cause the disk-like structures to rotate at observable frequencies. ${ }^{2}$

\section{Rotary Electrorheological Interactions}

Consider a monodispersed suspension of spherical particles of radius $r_{d}$ in an insulating fluid. For simplicity, we will assume that the particles have a simple dielectric response, so that their dipole moment obeys

$$
\vec{p}=\beta r_{d}^{3} \vec{E}
$$

where $\vec{E}$ is the instantaneous electric field. We will further simplify matters by considering only dipolar interactions between the particles; we ignore-non-electrostatic and multipolar interactions. As with ordinary ER fluids, we expect that this assumption will lead to a qualitatively correct and a quantitatively inadequate understanding of fluid behavior.

Let us suppose that although the frequency of the field $\omega$ is less than the characteristic frequency $\omega_{p}$ of the polarization response of the particles, that it is still greater than frequencies characteristic of particle movement in the fluid. To estimate these latter frequencies, we recall the Stokes relation for the steady velocity $\vec{v}$ of a particle of radius $r_{d}$ subjected to a constant force $\vec{F}$ in a fluid of viscosity $\mu_{0}{ }^{3}$

$$
\vec{F}=6 \pi \mu_{0} \vec{v} r_{d}
$$

Since we expect from dimensional analysis that the particles will be subjected to electrical forces $F_{E} \sim E^{2} r_{d}^{2}$, we conclude that the field frequency $\omega$ should obey

$$
\omega \gg \omega_{a}=\frac{\mu_{0}}{E^{2}}
$$

so that particles move a distance much less than their diameter in one cycle of the applied field. Note that elsewhere we have termed $\omega_{a}$ the aggregation frequency, since its inverse gives the characteristic time scale for particle aggregation in the ordinary ER effect. ${ }^{4}$

We have identified a range of frequencies $\omega_{a} \ll \omega \omega_{p}$ over which we can approximately regard the particles as stationary over one cycle of the applied field, but

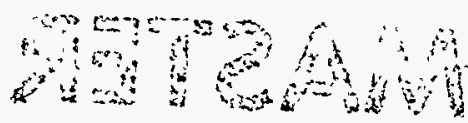


with dipole moments following the instantaneous field. In this regime, to find the effective interaction between particles, we should average the instantaneous interaction over one cycle of the applied field. The instantaneous interaction energy $u$ between two dipoles $\vec{p}^{(1)}$ and $\vec{p}^{(2)}$ separated by a distance $\vec{r}$ is

$$
u(\vec{r})=-p_{i}^{(1)}\left(\frac{3 r_{i} r_{j}-r^{2} \delta_{i j}}{r^{5}}\right) p_{j}^{(2)}
$$

where the indices are Cartesian. We suppose that $\vec{p}^{(1,2)}(t)=\beta r_{d}^{3} \vec{E}_{0}(t)$; thus the dipole moment is proportional to the applied field $\vec{E}_{0}(t)$-contributions of other dipoles to the local field are neglected. For a uniaxial field $\vec{E}_{0}(t)=\vec{E}_{0} f(t)$, the resulting averaged interaction is

$$
u(\vec{r})=-\frac{\dot{\beta}^{2} r_{d}^{6} E_{0}^{2}\left\langle f^{2}(t)\right\rangle}{r^{3}}\left(3 \cos ^{2} \theta-1\right)
$$

where the brackets \langle\rangle indicate a time average, and $\theta$ is the angle between $\vec{r}$ and the field direction. Equation 5 is simply the anisotropic dipolar interaction familiar from scores of ER fluid studies. We are concerned not with uniaxial fields, but with rotating fields

$$
\vec{E}_{0}(t)=E_{0}(\hat{x} \cos \omega t+\hat{y} \sin \omega t)
$$

where $\hat{x}$ and $\hat{y}$ are the normal three-dimensional basis vectors. Introducing this form for the field into Eq. (4) and averaging over time, we find that

$$
u(\vec{r})=-\frac{\beta^{2} r_{d}^{6}}{2 r^{3}}\left(1-3 \cos ^{2} \theta\right)
$$

where $\theta$ is now the angle between the direction perpendicular to the plane of the rotating electric feld and $\vec{r}$. Note that the sign of Eq. (7) is the opposite of that of Eq. (5). Thus the particles repel in the direction perpendicular to the plane of the field, and attract in the plane of the field. Just as the ordinary ER anisotropy lends itself to the formation of chains of particles, so the rotary ER anisotropy will lend itself to the formation of disks or sheets of particles; these disks are seen in experiment (see Figure 1.) 


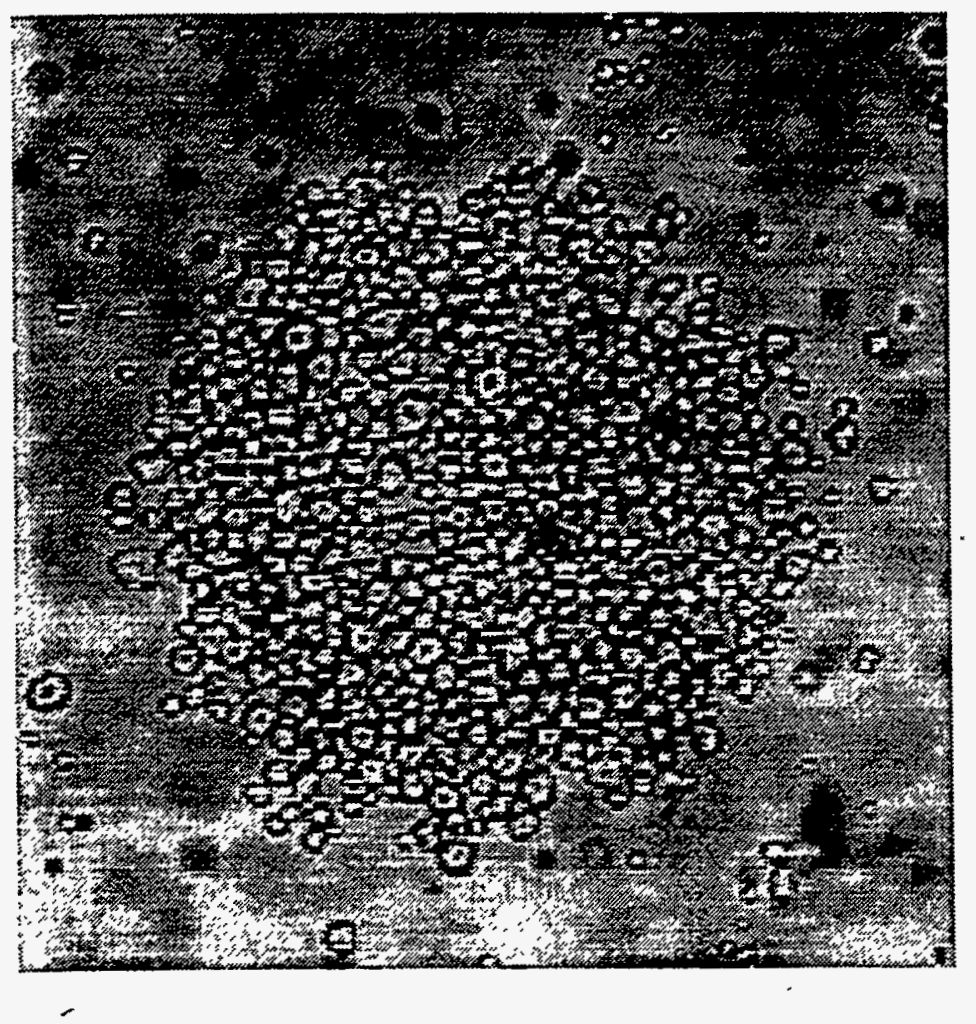

Figure 1. In a rotating electric field, particle disks form in the plane of the field. This photo shows a disk of $10 \mu \mathrm{m}$ corn starch particles in such a field. (The experimental results displayed in Figures 2 and 3 were obtained with $90 \mu \mathrm{m}$ corn pollen particles.)

\section{Electrical Torques and Disk Rotation}

One significant difference between the rotary ER effect and the ordinary ER effect is that significant torques develop on the particles in the former case. This effect is not to be confused with the torques that develop due to inter-particle interactions in, e.g., aqueous suspensions, as observed by Hu et al. ${ }^{5}$ The torque $T$ on a particle of dipole moment $\vec{p}$ in an applied field $\vec{E}_{0}$ is

$$
\vec{T}=\vec{p} \times \vec{E}_{0}
$$

Suppose that a particle is subjected to a uniaxial harmonic field $\vec{E}_{0} \cos (\omega t)$. If the polarization mechanism is linear, then the dipole moment of the particle will be $\vec{p}(t)=\beta(\omega) r_{d}^{3} \vec{E}_{0} \cos (\omega t-\delta(\omega))$, defining the polarizability $\beta$ and the phase shift $\delta$ as functions of frequency.' For the field given by Eq. (6), rotating at frequency $\omega$. it is clear that the dipole moment will be 


$$
\vec{p}(t)=\beta(\omega) r_{d}^{3} E_{0}(\hat{x} \cos (\omega t-\delta(\omega))+\hat{y} \sin (\omega t-\delta(\omega))
$$

and thus that the torque $\vec{T}$ will be

$$
\vec{T}=\hat{z} \beta(\omega) r_{d}^{3} E_{0}^{2} \sin \delta(\omega)
$$

For any polarization mechanism, we expect that the phase shift $\delta(\omega) \rightarrow 0$ for $\omega \rightarrow 0$. The behavior of $\beta(\omega \rightarrow 0)$ depends upon the nature of the polarization. If the effective dielectric contrast between the particle and the surrounding fluid is positive, then $\beta>0$, and the torque for small phase shift $\delta$ will tend to rotate the particle in the same sense as the applied field. On the other hand, if the effective dielectric contrast is negative, then $\beta<0$, and the torque will tend to rotate the particle in the opposite sense to that of the applied field.

As a concrete illustration of this latter case, consider an insulating particle suspended in a fluid of conductivity $\sigma$. For simplicity, we suppose that the particle and fluid can both be regarded as possessing underlying dielectric constants $\epsilon=1$, so we need consider only the conductive response of the fluid. Determining the dipole moment of the particle in an arbitrary applied field is now an elementary problem-current will flow in the fluid around the particle until the charge buildup at the particle surface is sufficient to cancel the normal electric field at the exterior particle surface (Gauss' law insures that there is an electric field interior to the particle in this case). A simple computation then shows that in this case

$$
\beta(\omega)=-\frac{1}{2} \frac{1}{1+(\omega / \sigma)^{2}}
$$

and

$$
\delta(\omega)=\tan ^{-1}(\omega / \sigma)
$$

where the $\omega \rightarrow 0$ result, $\beta=-1 / 2$, is that of a spherical hole in a medium of infinite dielectric constant.

A disk of particles, each subjected to a torque $\vec{T}$, will rotate at a frequency determined by the balance between this torque and the viscous drag. We model the disk as an oblate spheroid of semi-axes $(a, b, c)=\left(R, R, r_{d}\right)$, with $r_{d} \ll R$. The viscous torque $L$ on such a spheroid rotating at frequency $f$ in a fluid of viscosity $\mu_{0}$ is given (for sufficiently low Reynolds number) by ${ }^{6}$

$$
L=\frac{32}{3} \mu_{0} R^{3} f
$$


The electrical torque on this disk will be given by the torque per particle from Eq. (10) times the number of particles $N_{p}$. For simplicity we write $N_{p}=\kappa R^{2} / r_{d}^{2}$ with $\kappa \sim 1$, and obtain

$$
f \approx \frac{3 \kappa \beta(\omega) \sin \delta(\omega) E_{0}^{2}}{32 \mu_{0}}\left(\frac{r_{d}}{R}\right)
$$

Note that the frequency is considerably reduced below its naive dimensional value of $E_{0}^{2} / \mu_{0}$ by the factor $r_{d} / R$. This factor also insures that the viscous stresses at the edge of an isolated disk, no matter how large it is, are insufficient to cause yielding in the structure.

$90 \mu$ corn pollen

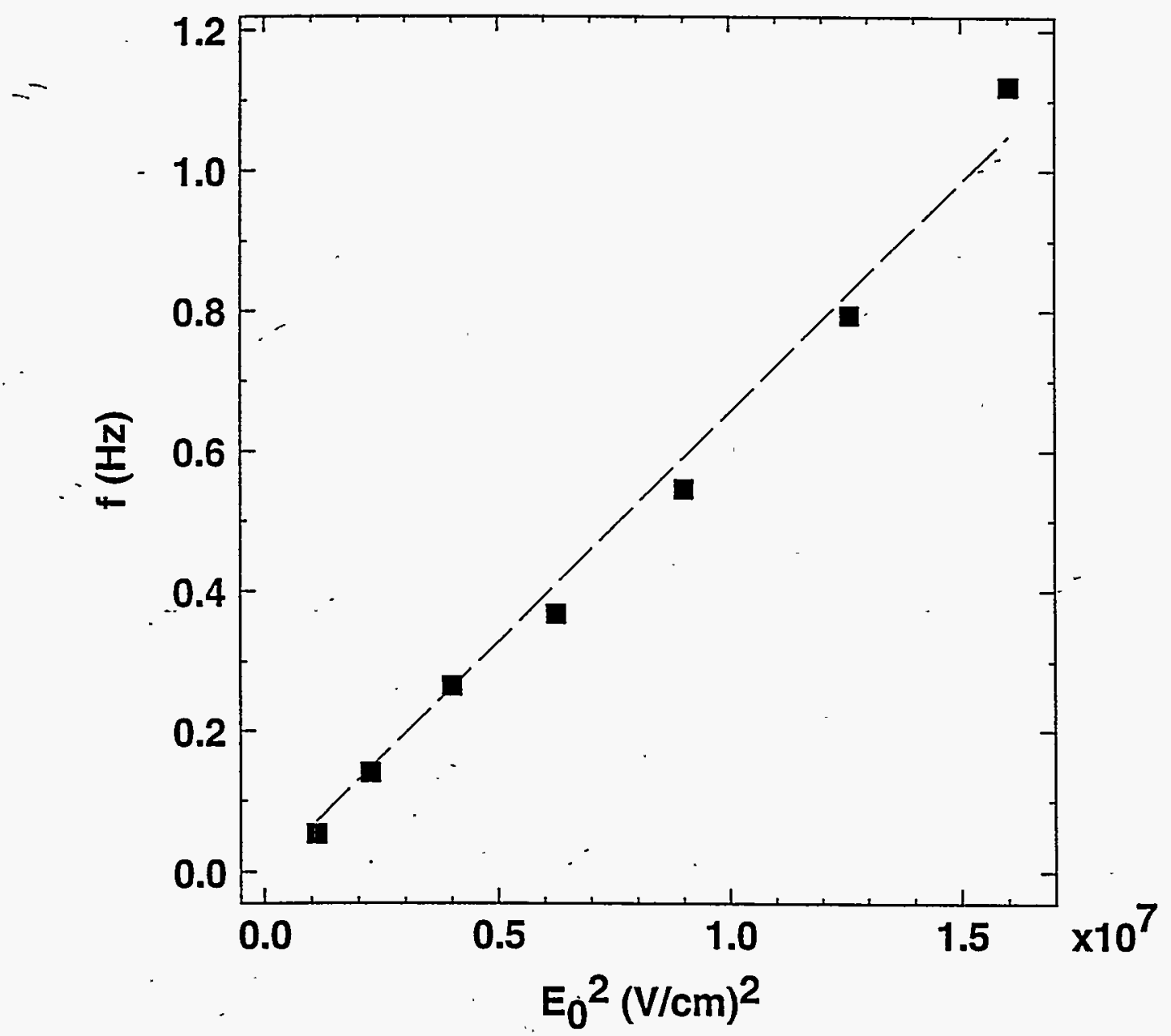

Figure 2. The disk rotation frequency $f$ versus field magnitude $E_{0}^{2}$ for $90 \mu \mathrm{m}$ corn pollen particles suspended in 4-methylcyclohexanol. The field rotates at a frequency of $1.0 \mathrm{kHz}$. Clearly $f \propto E_{0}^{2}$, as predicted by Eq. (13). 
$90 \mu$ corn pollen

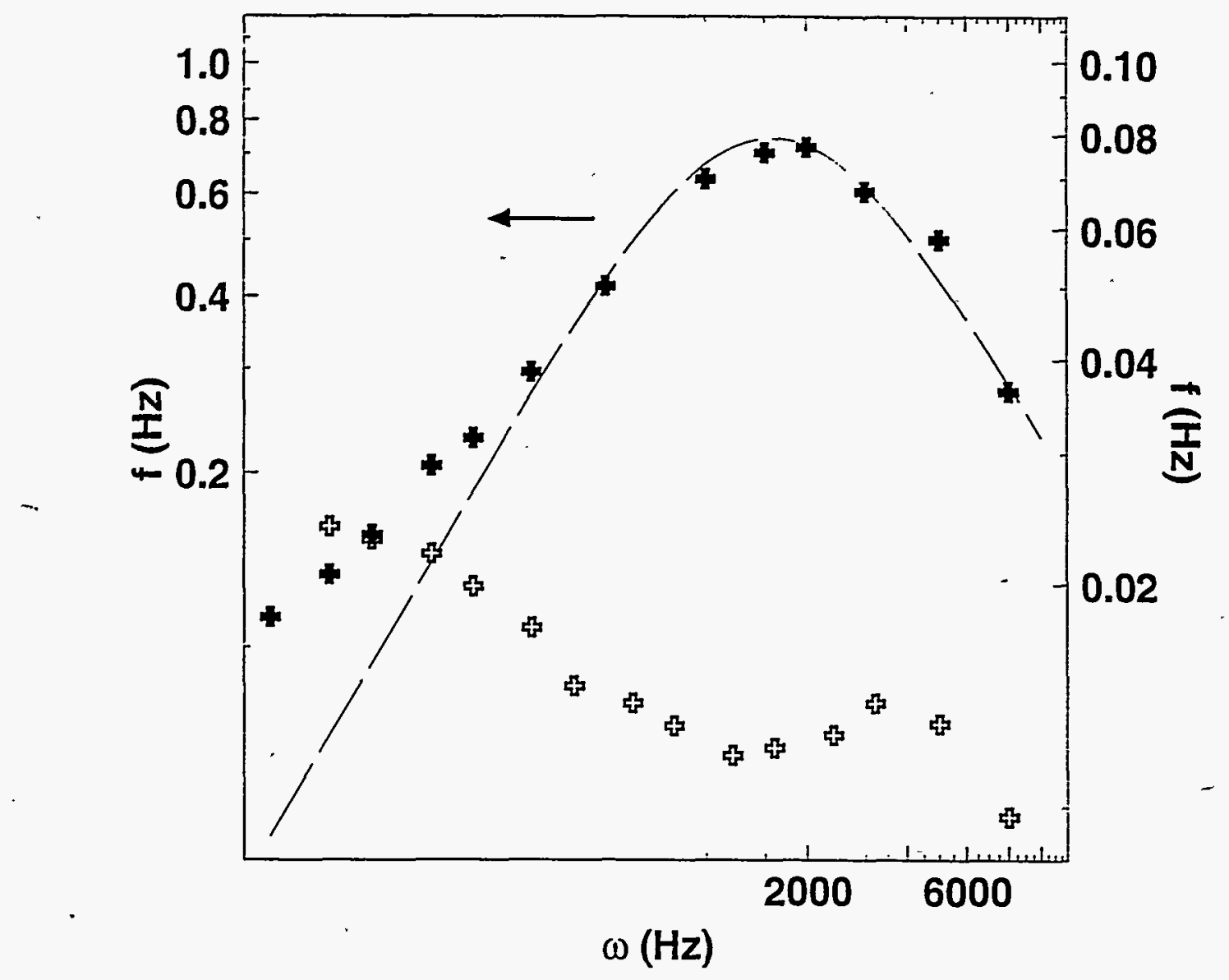

Figure 3. The disk rotation frequency $f$ versus field rotation frequency $\omega$ for 90 $\mu \mathrm{m}$ corn pollen particles suspended in 4-methylcyclohexanol (solid crosses, lefthand scale) or mineral oil (open crosses, right-hand scale) at a field magnitude of 4 $\mathrm{kV} / \mathrm{cm}$. The conducting solvent (4-methylcyclohexanol) results are well modelled by the Debye form from Eq. (14), with $\sigma=1.58 \times 10^{3} \mathrm{sec}^{-1}$ and $f_{0}=1.49 \mathrm{~Hz}$. The disk rotation in the mineral oil was an order of magnitude slower, with a more complicated frequency dispersion.

\section{Experimental Results}

We have tested these ideas by constructing a cell bounded by phased electrodes, which generate a spatially homogeneous but rotating electric field over a large region in the interior of the sample. Details of the experimental procedure and results will be published separately. ${ }^{7}$ Our ER fluid was made of $90 \mu \mathrm{m}$ corn pollen particles; these could be easily visualized. We suspended these particles either in the relatively conducting solvent 4-methylcyclohexanol or in a much more insulating mineral oil. 
In both solvents we were easily able to see disk formation at fields of from 0.3 to $4 \mathrm{kV} / \mathrm{cm}$, and at field rotation frequencies between 0.5 and $10 \mathrm{kHz}$. For the conducting solvent, the disks counter-rotated, opposite to the sense of rotation of the field. This agrees with the prediction in section 3 above regarding the behavior of insulating particles in a conducting solvent. Figure 3 shows the dependence of the disk rotation frequency $f$ on applied field; clearly the rotation frequency is $\propto E_{0}^{2}$, as predicted in Eq. (13).

The conducting model above predicts, using Eq. (11), that $f \propto \beta \sin \delta$, or equivalently that the dependence of $f$ on field frequency $\omega$ is

$$
f=f_{0} \frac{(\omega / \sigma)}{1+(\omega / \sigma)^{2}}
$$

Figure 3 shows the disk frequency $f$ versus the field frequency $\omega$ for each of the solvents. For the conducting solvent, a fit to the "Debye" form Eq. (14) is also shown; the agreement is good, although there is some additional dispersion at low frequencies. The insulating solvent behavior is not fit by a Debye form; in addition, the disks co-rotate with the field in these solvents, which suggests that the internal dielectric relaxation of the particles is determining the phase lag of the polarization in this case, leading to a positive effective dielectric contrast between the particles and the fluid.

\section{Acknowledgements}

- T.C.H. is grateful to the NSF for the support of this research through the PYI program, grant DMR-9057156. The work of R.A.A. and J.E.M. was performed at Sandia National Laboratories and was supported by the U.S. Department of Energy under Contract No.'DE-AC04-94AL85000.

\section{References}

1. See e.g. Electrorheological Fluids: Mechanisms, Properties, Technology, and Applications, eds. R. Tao and G.D. Roy, (World Scientific, Singapore, 1994).

2. There is a considerable literature on the torque experienced by a dielectric liquid in a circularly polarized field. See, e.g., E. Grossetti, Nuovo Cimento 10 (1958) $193 ; 13$ (1959) 350; and 19 (1961) 1.

3. L. Landäu and E.M. Lifshitz, Fluid Mechanics, (Pergamon, Oxford, 1959) pp. 63-68.

4. T.C. Halsey and W.R. Toor, Phys. Rev. Lett. 65 (1990) 2820; T.C. Halsey, Science 258 (1992) 761. 
5. Y. Hu, J.L. Glass, A.E. Griffith, and S. Fraden, J. Chem. Phys. 100 (1994) 4674.

6. G.B. Jeffery, Proc. Roy. Soc. A102 (1922) 761.

7. R.A. Anderson, T.C. Halsey, and J.E. Martin, unpublished. 
$\therefore$ 